\title{
$B$ anomalies in the nonminimal universal extra dimension model
}

\author{
Jong-Phil Lee๑* \\ Sang-Huh College, Konkuk University, Seoul 05029, Korea
}

(Received 19 June 2019; published 2 October 2019)

\begin{abstract}
We investigate $B$ anomalies in the framework of the nonminimal universal extra dimension model. Newly measured polarization parameters in $B \rightarrow D^{(*)} \tau \nu, P_{\tau}\left(D^{(*)}\right)$, and $F_{L}\left(D^{*}\right)$ as well as the ratios $R\left(D^{(*)}\right)$ are considered altogether. The Kaluza-Klein modes of the $W$ boson and charged scalar contributes to the new physics effects. We find that the model parameters fit the global data very well with the minimum $\chi^{2} /$ d.o.f. near unity, rendering $B_{c} \rightarrow \tau \nu$ branching ratios to be a few percent. The best-fit values of $R(D)$ and $R\left(D^{*}\right)$ are still far from $(\gtrsim 2 \sigma)$ the standard model predictions.
\end{abstract}

DOI: 10.1103/PhysRevD.100.075005

\section{INTRODUCTION}

The standard model (SM) of particle physics has been up to now very successful in explaining many phenomena in our Universe. The last missing piece of the SM, the Higgs particle, was finally discovered in 2012. But there must be some new physics (NP) beyond the SM. Flavor physics is a good testing ground for the NP. Recently, some anomalies were reported in $b \rightarrow c$ semileptonic decays. The fraction of the branching ratios

$$
R\left(D^{(*)}\right) \equiv \frac{\operatorname{Br}\left(B \rightarrow D^{(*)} \tau \nu\right)}{\operatorname{Br}\left(B \rightarrow D^{(*)} \ell \nu\right)}
$$

reveals an excess over the SM predictions [1],

$$
\begin{aligned}
R(D)_{\mathrm{SM}} & =0.299 \pm 0.003, \\
R\left(D^{*}\right)_{\mathrm{SM}} & =0.258 \pm 0.005 .
\end{aligned}
$$

Experiments including $B A B A R$, Belle, and LHCb have reported somewhat larger values of $R\left(D^{(*)}\right)$ than those of Eq. (2) by about $2 \sigma \sim 3 \sigma$ [2-11]. Recently, the Belle Collaboration announced new results [9],

$$
\begin{aligned}
R(D)_{\text {Belle1904 }} & =0.307 \pm 0.037 \pm 0.016, \\
R\left(D^{*}\right)_{\text {Belle1904 }} & =0.283 \pm 0.018 \pm 0.014,
\end{aligned}
$$

which are closer to Eq. (2) than the previous data and consistent with the SM within $1.2 \sigma$. Combined results for

\footnotetext{
*jongphil7@gmail.com
}

Published by the American Physical Society under the terms of the Creative Commons Attribution 4.0 International license. Further distribution of this work must maintain attribution to the author(s) and the published article's title, journal citation, and DOI. Funded by SCOAP. all data by the Heavy Flavor Averaging Group (HFLAV) Collaboration [12],

$$
\begin{aligned}
R(D)_{\mathrm{HFLAV}} & =0.340 \pm 0.027 \pm 0.013 \\
R\left(D^{*}\right)_{\mathrm{HFLAV}} & =0.295 \pm 0.011 \pm 0.008
\end{aligned}
$$

give a discrepancy between the SM predictions and experimental data at the $3.08 \sigma$ level. The BABAR measurements $[2,3]$ exclude at the $99.8 \%$ confidence level the type-II two-Higgs-doublet model (2HDM) where a charged Higgs boson contributes to $R\left(D^{(*)}\right)$, while the Belle measurements [4] are compatible with the type-II 2HDM. It was shown that an anomalous $\tau$ coupling to the charged Higgs in the 2HDM can explain the data very well [13]. In extra dimension models the overlapping between the wave functions of $\tau$ and the neutral scalar could be weak to make $\tau$ screened from the scalar vacuum, resulting in an enhancement of $\tau$ couplings to charged Higgs. For discussions in the 2HDM, see Refs. [14-19]. There are many other NP scenarios to explain the $R\left(D^{(*)}\right)$ anomaly, including leptoquark models [20-26], composite models [27-30], warped extra dimensions [31-34], etc. [35-37].

On top of the ratio $R\left(D^{(*)}\right)$ the Belle Collaboration measured the relevant polarizations in $B \rightarrow D^{(*)} \tau \nu$ decays. One can consider observable parameters associated with $D^{*}$ as well as $\tau$. The $\tau$-polarization asymmetry is defined as

$$
P_{\tau}\left(D^{(*)}\right) \equiv \frac{\Gamma_{\tau}^{D^{(*)}}(+)-\Gamma_{\tau}^{D^{(*)}}(-)}{\Gamma_{\tau}^{D^{(*)}}(+)+\Gamma_{\tau}^{D^{(*)}}(-)},
$$

where $\Gamma_{\tau}^{D^{(*)}}( \pm)$ is the decay width for $( \pm) \tau$ helicity. The SM predictions are $[38,39]$ 


$$
\begin{aligned}
P_{\tau}(D)_{\mathrm{SM}} & =0.325 \pm 0.009, \\
P_{\tau}\left(D^{*}\right)_{\mathrm{SM}} & =-0.497 \pm 0.013 .
\end{aligned}
$$

The experimental result is $[7,8]$

$$
P_{\tau}\left(D^{*}\right)=-0.38 \pm 0.51_{-0.16}^{+0.21} .
$$

The longitudinal $D^{*}$ polarization is

$$
F_{L}\left(D^{*}\right) \equiv \frac{\Gamma\left(B \rightarrow D_{L}^{*} \tau \nu\right)}{\Gamma\left(B \rightarrow D^{*} \tau \nu\right)},
$$

where the Belle's measurement is [40]

$$
F_{L}\left(D^{*}\right)=0.60 \pm 0.08 \pm 0.035,
$$

while the SM value is estimated to be [41]

$$
F_{L}\left(D^{*}\right)_{\mathrm{SM}}=0.46 \pm 0.04
$$

The polarization parameters could provide more information about the Lorentz structure of possible NP.

In this paper we consider the nonminimal universal extra dimension (NMUED) model [42-49] to fit the global data on $R\left(D^{(*)}\right)$ and polarization parameters. In the universal extra dimension (UED) models there is an extra spacelike dimension with a flat metric compactified on an $S^{1} / Z_{2}$ orbifold, where the SM particles could reside. Each SM particles is accompanied by infinite towers of Kaluza-Klein (KK) states. There are two branes at the end points of the orbifold. The reflection symmetry of the bulk space provides with the KK-parity conservation. The lightest KK particle is a natural candidate for dark matter, which makes the UED scenario a strong alternative to the SM. As discussed in Ref. [50], in the minimal version of the UED (MUED) there are no new couplings at the tree level relevant to $R\left(D^{(*)}\right)$. The radiative corrections include bulk corrections and boundary localized ones. In the MUED models the latter is adjusted to cancel the cutoff dependent corrections. The NMUED models allow the boundary localized terms (BLTs) to be free parameters. In this analysis we include the BLTs with free strength parameters. The presence of BLTs changes mass spectrum and couplings of KK modes of the UED model. The NP effects enter through the possible interactions between a pair of zero-mode fermion and even KK modes of charged gauge boson or scalar associated with the BLTs [50,51]. These kinds of interactions are not allowed in the MUED because of the KK wave function orthogonality. Since the new interactions contribute to $R\left(D^{(*)}\right)$ at tree level, we expect the NMUED model would provide some hints to solve the $R\left(D^{(*)}\right)$ puzzle.

The paper is organized as follows. In Sec. II the NMUED model is introduced. Section III provides the various observables in numerical forms. The results and discussions are given in Sec. IV, and we conclude in Sec. V.

\section{NMUED MODEL}

We assume that there is one flat extra dimension $(y)$ compactified on an $S^{1} / Z_{2}$ orbifold with radius $R$. Two branes are located at the end points $y=0$ and $y=\pi R$ where both boundary terms are equal. The five-dimensional (5D) action for fermions $f$ is [50]

$$
\begin{aligned}
\mathcal{S}_{f}= & \sum_{f=q, \ell} \int d^{4} x \int_{0}^{\pi R} d y\left\{i \bar{\Psi}_{L}^{f} \Gamma^{M} \mathcal{D}_{M} \Psi_{L}^{f}\right. \\
& +r_{f}[\delta(y)+\delta(y-\pi R)] i \bar{\Psi}_{L}^{f} \gamma^{\mu} \mathcal{D}_{\mu} P_{L} \Psi_{L}^{f}+i \bar{\Psi}_{R}^{f} \Gamma^{M} \mathcal{D}_{M} \Psi_{R}^{f} \\
& \left.+r_{f}[\delta(y)+\delta(y-\pi R)] i \bar{\Psi}_{R} \gamma^{\mu} \mathcal{D}_{\mu} P_{R} \Psi_{R}^{f}\right\},
\end{aligned}
$$

where $\Psi_{L, R}^{f}(x, y)$ are the 5D four component Dirac spinors for fermions $f=q, \ell$. In terms of two component spinors,

$\Psi_{L, R}^{f}(x, y)=\left(\begin{array}{c}\psi_{L, R}^{f}(x, y) \\ \chi_{L, R}^{f}(x, y)\end{array}\right)=\sum_{n}\left(\begin{array}{c}\psi_{L, R}^{f(n)}(x) F_{L, R}^{f(n)}(y) \\ \chi_{L, R}^{f(n)}(x) G_{L, R}^{f(n)}(y)\end{array}\right)$,

where $F_{L, R}^{f(n)}(y)$ and $G_{L, R}^{f(n)}(y)$ are the $n$th KK wave functions. In Eq. (11) $r_{f}$ is the strength of the boundary localized terms. They are related to the mass of the $n$th KK excitation $m_{f^{(n)}}$ by the transcendental equation

$$
\frac{r_{f} m_{f^{(n)}}}{2}= \begin{cases}-\tan \left(\frac{m_{f^{(n)}} \pi R}{2}\right) & \text { for even } n, \\ \cot \left(\frac{m_{f^{(n)}} \pi R}{2}\right) & \text { for odd } n .\end{cases}
$$

As for the gauge boson sector, the 5D action is

$$
\begin{aligned}
\mathcal{S}_{\text {gauge }}= & -\frac{1}{4} \int d^{4} x \int_{0}^{\pi R} d y\left\{W_{M N}^{i} W^{i M N}\right. \\
& +r_{V}[\delta(y)+\delta(y-\pi R)] W_{\mu \nu}^{i} W^{i \mu \nu} \\
& \left.+B_{M N} B^{M N}+r_{V}[\delta(y)+\delta(y-\pi R)] B_{\mu \nu} B^{\mu \nu}\right\},
\end{aligned}
$$

where $W_{M N}^{i}, B_{M N}$ are the 5D gauge field strength tensors. The $n$th KK mass of the gauge boson is

$$
M_{W^{(n)}}=\sqrt{M_{W}^{2}+m_{V^{(n)}}^{2}},
$$

where $m_{V^{(n)}}$ satisfies the same transcendental equation as Eq. (13). For the 5D scalar field $\Phi(x, y)$, the action is 


$$
\begin{aligned}
\mathcal{S}_{\phi}= & \int d^{4} x \int_{0}^{\pi R} d y\left\{\left(\mathcal{D}_{M} \Phi\right)^{\dagger}\left(\mathcal{D}^{M} \Phi\right)\right. \\
& \left.+r_{\phi}[\delta(y)+\delta(y-\pi R)]\left(\mathcal{D}_{\mu} \Phi\right)^{\dagger}\left(\mathcal{D}^{\mu} \Phi\right)\right\} .
\end{aligned}
$$

We choose $r_{\phi}=r_{V}$ for proper gauge fixing [52], and consequently the mass of the KK scalar is $m_{\phi^{(n)}}=m_{V^{(n)}}$. The Yukawa interaction is described by

$$
\begin{aligned}
\mathcal{S}_{Y}= & -\sum_{f} \int d^{4} x \int_{0}^{\pi R} d y\left\{\lambda_{5} \bar{\Psi}_{L}^{f} \tilde{\Phi} \Psi_{R}^{f}\right. \\
& \left.+r_{Y}[\delta(y)+\delta(y-\pi R)] \lambda_{5} \bar{\psi}^{f}{ }_{L} \tilde{\Phi}_{R}^{f}+\text { H.c. }\right\},
\end{aligned}
$$

where $\lambda_{5}$ is the 5D Yukawa coupling and $r_{Y}$ is the boundary strength.

In NMUED new KK particles contribute to $B$ decays. As mentioned in Sec. I, even KK modes of the $W$ boson as well as the charged Higgs couple to a pair of zero-mode fermions, which provide new vector and scalar interactions, respectively. The effects are encoded in the overlap integrals

$$
\begin{aligned}
I_{n}^{f g}= & \sqrt{\pi R\left(1+\frac{r_{V}}{\pi R}\right)} \\
& \times \int_{0}^{\pi R} d y\left\{1+r_{f}[\delta(y)+\delta(y-\pi R)]\right\} a^{n} F_{L}^{f(0)} F_{L}^{f(0)}, \\
I_{n}^{f Y}= & \sqrt{\pi R\left(1+\frac{r_{V}}{\pi R}\right)} \\
& \times \int_{0}^{\pi R} d y\left\{1+r_{Y}[\delta(y)+\delta(y-\pi R)]\right\} f^{n} F_{L}^{f(0)} G_{R}^{f(0)},
\end{aligned}
$$

where $a^{n}$ and $h^{n}$ are $n$th KK mode of the $W$ boson and scalar, respectively. For $r_{\phi}=r_{V}$ and $a^{n}=h^{n}$, and further if $r_{f}=r_{Y}$, then [50]

$$
I_{n}^{f g}=I_{n}^{f Y} \equiv I_{n}^{f}=\frac{\sqrt{2}\left(\hat{r}_{f}-\hat{r}_{V}\right) \sqrt{1+\hat{r}_{V}}}{\left(1+\hat{r}_{f}\right) \sqrt{1+r_{V}^{2} m_{V^{(n)}}^{2} / 4+\hat{r}_{V}}},
$$

where $\hat{r} \equiv r /(\pi R)$. Actually, $I_{n}^{f}$ is the interaction term between a pair of zero-mode fermion $f$ and $n$th KK modes of $W$ boson or scalar, which encodes the NP effects on observables.

\section{OBSERVABLES}

Now the effective Hamiltonian for $b \rightarrow c \ell \nu$ is

$$
\mathcal{H}_{\text {eff }}=\frac{4 G_{F}}{\sqrt{2}} V_{c b} \sum_{\ell=\mu, \tau}\left\{\left(1+C_{V}^{\ell}\right) \mathcal{O}_{V}^{\ell}+C_{S}^{\ell} \mathcal{O}_{S}^{\ell}\right\},
$$

where the operators $\mathcal{O}_{V, S}^{\ell}$ are defined by

$$
\begin{gathered}
\mathcal{O}_{V}^{\ell}=\left(\bar{c}_{L} \gamma^{\mu} b_{L}\right)\left(\bar{\ell}_{L} \gamma_{\mu} \nu_{\ell L}\right), \\
\mathcal{O}_{S}^{\ell}=\left(\bar{c}_{L} b_{R}\right)\left(\bar{\ell}_{R} \nu_{\ell L}\right) .
\end{gathered}
$$

The NP effects are encapsulated in the Wilson coefficients $C_{V, S}^{\ell}$ given as [50]

$$
\begin{gathered}
C_{V}^{\ell}=\sum_{n \geq 2}\left[\frac{M_{W}^{2}}{M_{W^{(n)}}^{2}}\right] I_{n}^{q} I_{n}^{\ell}, \\
C_{S}^{\ell}=\sum_{n \geq 2}\left[\frac{m_{b} m_{\ell}}{M_{W^{(n)}}^{2}}\right]\left[\frac{M_{W}^{2}}{M_{W^{(n)}}^{2}}\right]\left\{\operatorname { c o s } \left(\frac{1}{2} \tan ^{-1}\left[\frac{m_{c}}{m_{f^{(n)}}}\right]\right.\right. \\
\left.-\frac{1}{2} \tan ^{-1}\left[\frac{m_{\ell}}{m_{f^{(n)}}}\right]\right) \\
\left.-\sin \left(\frac{1}{2} \tan ^{-1}\left[\frac{m_{c}}{m_{f^{(n)}}}\right]+\frac{1}{2} \tan ^{-1}\left[\frac{m_{\ell}}{m_{f^{(n)}}}\right]\right)\right\} I_{n}^{q} I_{n}^{\ell} .
\end{gathered}
$$

From $\mathcal{H}_{\text {eff }}$ one can calculate the transition amplitudes and decay rates for $B \rightarrow D^{(*)}$ decays and can construct various observable parameters. We concentrate only on the numerical results for the observables in our analysis. Numerically the observables for $B \rightarrow D^{(*)} \ell \nu_{\ell}$ decays are (at $\mu=m_{b}$ scale) [53]

$$
\begin{gathered}
R(D)=2 R_{\mathrm{SM}}(D) \frac{\left(1+C_{V}^{\tau}\right)^{2}+1.54\left(1+C_{V}^{\tau}\right) C_{S}^{\tau}+1.09\left(C_{S}^{\tau}\right)^{2}}{1+\left(1+C_{V}^{\mu}\right)^{2}+1.54\left(1+C_{V}^{\mu}\right) C_{S}^{\mu}+1.09\left(C_{S}^{\mu}\right)^{2}} \\
R\left(D^{*}\right)=2 R_{\mathrm{SM}}\left(D^{*}\right) \frac{\left(1+C_{V}^{\tau}\right)^{2}+0.13\left(1+C_{V}^{\tau}\right) C_{S}^{\tau}+0.05\left(C_{S}^{\tau}\right)^{2}}{1+\left(1+C_{V}^{\mu}\right)^{2}+0.13\left(1+C_{V}^{\mu}\right) C_{S}^{\mu}+0.05\left(C_{S}^{\mu}\right)^{2}} \\
P_{\tau}(D)=\frac{0.32\left(1+C_{V}^{\tau}\right)^{2}+1.54\left(1+C_{V}^{\tau}\right) C_{S}^{\tau}+1.09\left(C_{S}^{\tau}\right)^{2}}{\left(1+C_{V}^{\tau}\right)^{2}+1.54\left(1+C_{V}^{\tau}\right) C_{S}^{\tau}+1.09\left(C_{S}^{\tau}\right)^{2}}
\end{gathered}
$$


TABLE I. Experimental data for $R\left(D^{(*)}\right), \quad P_{\tau}\left(D^{(*)}\right)$, and $F_{L}\left(D^{*}\right)$. The uncertainties are \pm (statistical) \pm (systematic). For the third uncertainty of $\operatorname{LHCb}(1711)$, see Ref. [11] for details. For BABAR, Belle(2015), and Belle(2019) results, the correlations between $R(D)$ and $R\left(D^{*}\right)$ are $-0.31,-0.50$, and -0.51 , respectively [12].

\begin{tabular}{lcc}
\hline \hline & $R(D)$ & $R\left(D^{*}\right)$ \\
\hline BABAR & $0.440 \pm 0.058 \pm 0.042$ & $0.332 \pm 0.024 \pm 0.018[3]$ \\
Belle(2015) & $0.375 \pm 0.064 \pm 0.026$ & $0.293 \pm 0.038 \pm 0.015[4]$ \\
Belle(2016) & $\ldots$ & $0.302 \pm 0.030 \pm 0.011[5]$ \\
Belle(2017) & $\ldots$ & $0.276 \pm 0.034_{-0.026}^{+0.029}[6]$ \\
Belle(2017) & $\ldots$ & $0.270 \pm 0.035_{-0.025}^{+0.028}[7,8]$ \\
Belle(2019) & $0.307 \pm 0.037 \pm 0.016$ & $0.283 \pm 0.018 \pm 0.014[9]$ \\
LHCb(2015) & $\ldots$ & $0.336 \pm 0.027 \pm 0.030[10]$ \\
LHCb(2017) & $\ldots$ & $0.291 \pm 0.019 \pm 0.026 \pm 0.013[11]$ \\
Belle(2017) & $P_{\tau}\left(D^{*}\right)$ & $F_{L}\left(D^{*}\right)$ \\
Belle(2019) & $\ldots .0 .16[7,8]$ & $\cdots$ \\
\hline \hline
\end{tabular}

$$
\begin{gathered}
P_{\tau}\left(D^{*}\right)=\frac{-0.49\left(1+C_{V}^{\tau}\right)^{2}+0.13\left(1+C_{V}^{\tau}\right) C_{S}^{\tau}+0.05\left(C_{S}^{\tau}\right)^{2}}{\left(1+C_{V}^{\tau}\right)^{2}+0.13\left(1+C_{V}^{\tau}\right) C_{S}^{\tau}+0.05\left(C_{S}^{\tau}\right)^{2}}, \\
F_{L}\left(D^{*}\right)=\frac{0.46\left(1+C_{V}^{\tau}\right)^{2}+0.13\left(1+C_{V}^{\tau}\right) C_{S}^{\tau}+0.05\left(C_{S}^{\tau}\right)^{2}}{\left(1+C_{V}^{\tau}\right)^{2}+0.13\left(1+C_{V}^{\tau}\right) C_{S}^{\tau}+0.05\left(C_{S}^{\tau}\right)^{2}}, \\
\operatorname{Br}\left(B_{c} \rightarrow \tau \nu\right)=0.02\left(\frac{f_{B_{c}}}{0.43 \mathrm{GeV}}\right)\left[1+C_{V}^{\tau}+4.3 C_{S}^{\tau}\right]^{2} .
\end{gathered}
$$

The results are obtained from the numerical values of the relevant form factors of $B \rightarrow D$ [54] and $B \rightarrow D^{*}$ transitions [1,55].

The branching ratio of $B_{c} \rightarrow \tau \nu, \operatorname{Br}\left(B_{c} \rightarrow \tau \nu\right)$ could impose strong constraints on $R\left(D^{(*)}\right)$ [56]. Since $\operatorname{Br}\left(B_{c} \rightarrow \tau \nu\right) \sim\left(1+C_{V}^{\tau}+4.3 C_{S}^{\tau}\right)^{2}$, the branching ratio directly affects the relevant Wilson coefficients. There are still debates on the upper bound of $\operatorname{Br}\left(B_{c} \rightarrow \tau \nu\right)$. The strongest bound is from Ref. [57], where $\operatorname{Br}\left(B_{c} \rightarrow \tau \nu\right)<10 \%$. On the other hand, Ref. [53] argues that the branching ratio could be as large as $60 \%$. In this analysis we do not explicitly impose the $\operatorname{Br}\left(B_{c} \rightarrow \tau \nu\right)$ constraints because, as we will see later, our results are compatible with small values of $\operatorname{Br}\left(B_{c} \rightarrow \tau \nu\right)$. The experimental data for various observables used in this analysis are listed in Table I.

\section{RESULTS}

We implement the global $\chi^{2}$ fit for the observables in Table I. We first define $\chi^{2}$ as

$$
\chi^{2} \equiv \sum_{i, j}\left[\mathcal{O}_{i}^{\exp }-\mathcal{O}_{i}^{\text {th }}\right] \mathcal{C}_{i j}^{-1}\left[\mathcal{O}_{j}^{\text {exp }}-\mathcal{O}_{j}^{\text {th }}\right]
$$

where $\mathcal{O}_{i}^{\text {exp }}$ are the experimental data, while $\mathcal{O}_{i}^{\text {th }}$ are the theoretical predictions of Eqs. (25)-(30), and $\mathcal{C}_{i j}$ are the correlation matrix elements.

There are two major constraints. One is from the oblique parameters of the electroweak precision test (EWPT) [58-61]. In the NMUED model the Fermi constant is modified by the tree-level contributions of even $n$th $\mathrm{KK}$ modes of $W$ bosons to the four-fermion interactions. This kind of correction is absent in the MUED scenario. The Fermi constant in NMUED is now written as

$$
G_{F}=G_{F}^{0}+\delta G_{F}
$$

Here $G_{F}^{0}$ is the Fermi constant in the SM and $\delta G_{F}$ is the correction from the new contributions of the $W^{ \pm} \mathrm{KK}$ modes. Explicitly [50],

$$
G_{F}^{0}=\frac{g^{2}}{4 \sqrt{2} M_{W}^{2}}, \quad \delta G_{F}=\sum_{n \geq 2} \frac{g^{2}\left(I_{n}^{\ell}\right)^{2}}{4 \sqrt{2} m_{W^{(n)}}^{2}},
$$

where $g$ is the gauge coupling constant. Note that $\delta G_{F} \sim$ $\left(I_{n}^{\ell}\right)^{2}$ because the Fermi constant is derived from the muon lifetime. We consider only the second KK contributions for simplicity. Now the Fermi constant is related to the PeskinTakeuchi parameters as [58] 
TABLE II. Best-fit values.

\begin{tabular}{ccccccc}
\hline \hline$R(D)$ & $R\left(D^{*}\right)$ & $P_{\tau}(D)$ & $P_{\tau}\left(D^{*}\right)$ & $F_{L}\left(D^{*}\right)$ & $\operatorname{Br}\left(B_{c} \rightarrow \tau \nu\right)$ & $\chi_{\min }^{2} /$ d.o.f. \\
\hline 0.343 & 0.296 & 0.320 & -0.490 & 0.460 & $2.75 \times 10^{-2}$ & 1.25 \\
\hline
\end{tabular}

$$
\begin{aligned}
S_{\mathrm{NMUED}} & =0, \quad T_{\mathrm{NMUED}}=-\frac{1}{\alpha} \frac{\delta G_{F}}{G_{F}}, \\
U_{\mathrm{NMUED}} & =\frac{4 \sin ^{2} \theta_{W}}{\alpha} \frac{\delta G_{F}}{G_{F}},
\end{aligned}
$$

where we neglect possible loop effects which are subdominant compared to the tree-level contributions to $\delta G_{F}$. We use the data [62]

$S=0.05 \pm 0.11, T=0.09 \pm 0.13, \quad U=0.01 \pm 0.11$,

where the correlation coefficients are

$\rho_{S T}=0.90, \quad \rho_{T U}=-0.83, \quad \rho_{U S}=-0.59$.

Following the methods of Ref. [61], we impose the $S, T, U$ constraints by requiring $\chi_{S T U}^{2}<6.18$ at $2 \sigma$, where $\chi_{S T U}^{2}$ is defined by the covariant matrix relevant for the $S, T, U$ parameters, as in Eq. (31).

The other major constraint comes from the LHC dilepton resonance searches. At the LHC the second KK gauge boson $A^{(2)}$ can be produced via the KK number violating

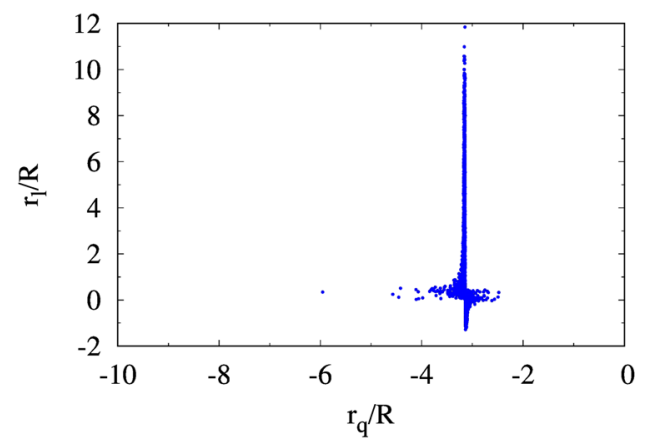

(a)

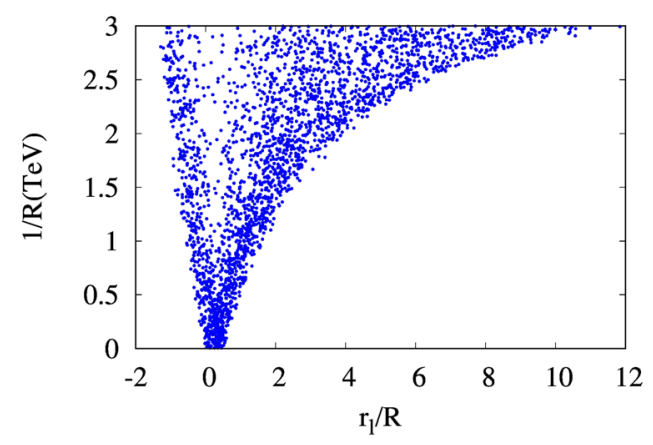

(c) interactions, subsequently decaying into the SM particles. Recent results from ATLAS dilepton resonance searches at $13 \mathrm{TeV}$ with $13.3 \mathrm{fb}^{-1}$ provide a stringent constraint on the NMUED parameters [63]. We reflect the results of Ref. [63] on the strength of the BLT in the gauge sector to constrain our analysis to the region $0 \leq r_{V} / R \leq 0.5$. The best-fit values for the minimum $\chi^{2}$ are listed in Table II.

In Fig. 1 we plot the allowed regions of the NMUED parameters at the $2 \sigma$ level. We scanned over the range $0 \leq 1 / R \leq 3 \mathrm{TeV}$. A noticeable feature is that the allowed range of $r_{q} / R$ is rather narrow with negative values, contrary to that of $r_{\ell} / R$, as shown in Fig. 1(a). In the NMUED models, $r_{q, \ell}$ are considered as free parameters and can be negative, but with some restrictions. The fields $F_{L, R}^{f(n)}$ and $G_{L, R}^{f(n)}$ of Eq. (12) have normalization factor [48-50]

$$
N_{n}^{f}=\sqrt{\frac{2}{\pi R}} \frac{1}{\sqrt{1+r_{f}^{2} m_{f^{(n)}}^{2} / 4+r_{f} /(\pi R)}} .
$$

For $N_{n}^{f}$ to be meaningful, $r_{f} /(\pi R)>-1-r_{f}^{2} m_{f^{(n)}}^{2} / 4$ and, for small values of $r_{f} m_{f^{(n)}}, r_{f} / R \gtrsim-\pi$. Our results in Fig. 1 satisfy this requirement. Note that the points near $r_{q} / R=$ $-\pi$ are favorable for larger $R(D)$ and smaller $\chi^{2}$.

Figure 2 shows the second KK masses $m_{W^{(2)}}$ and $m_{\tau^{(2)}}$. Allowed values of various observables at $2 \sigma$ are given in

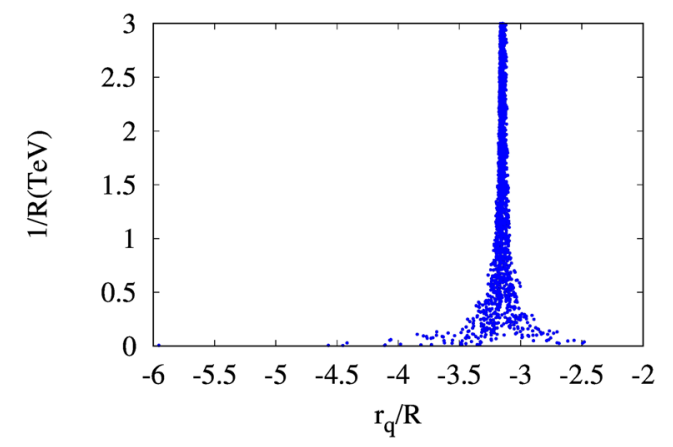

(b)

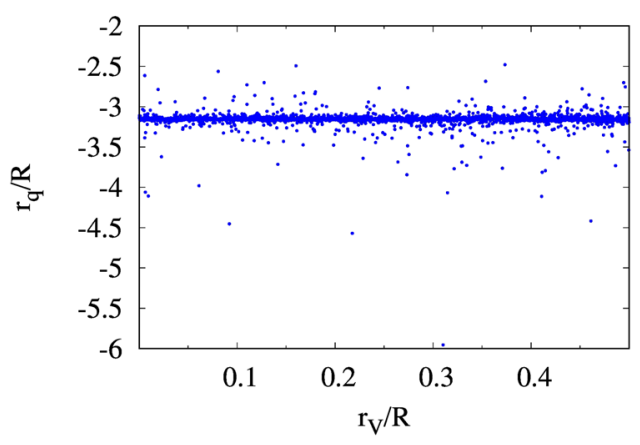

(d)

FIG. 1. Allowed regions of model parameters of NMUED at the $2 \sigma$ level. (a) $r_{\ell} / R$ vs $r_{q} / R$; (b) $1 / R$ (TeV) vs $r_{q} / R$; (c) $1 / R$ (TeV) vs $r_{\ell} / R ;$ (d) $r_{q} / R$ vs $r_{V} / R$. 


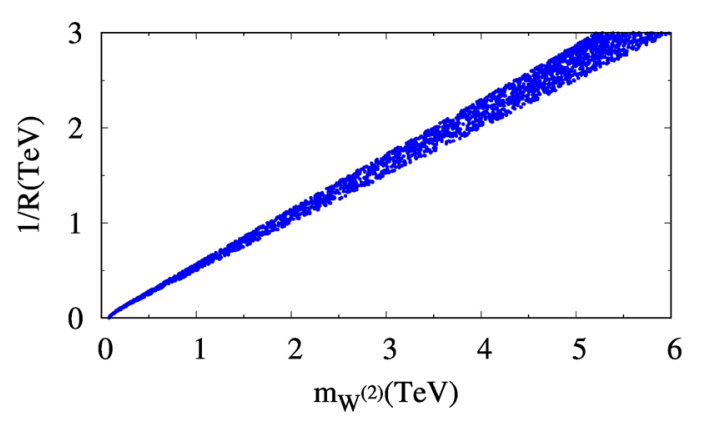

(a)

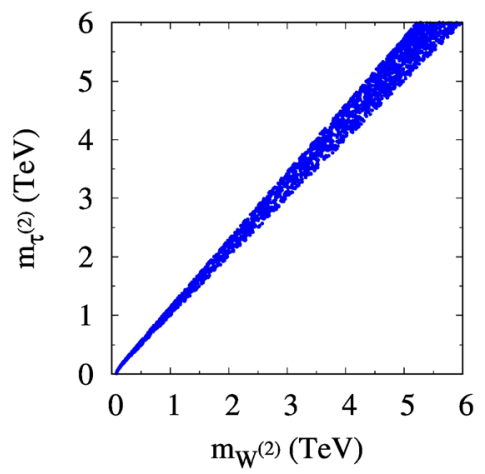

(b)

FIG. 2. Mass scales of NMUED at $2 \sigma$. (a) $1 / R(\mathrm{TeV})$ vs $m_{W^{(2)}}(\mathrm{TeV}) ;\left(\right.$ b) $m_{\tau^{(2)}}(\mathrm{TeV})$ vs $m_{W^{(2)}}(\mathrm{TeV})$.

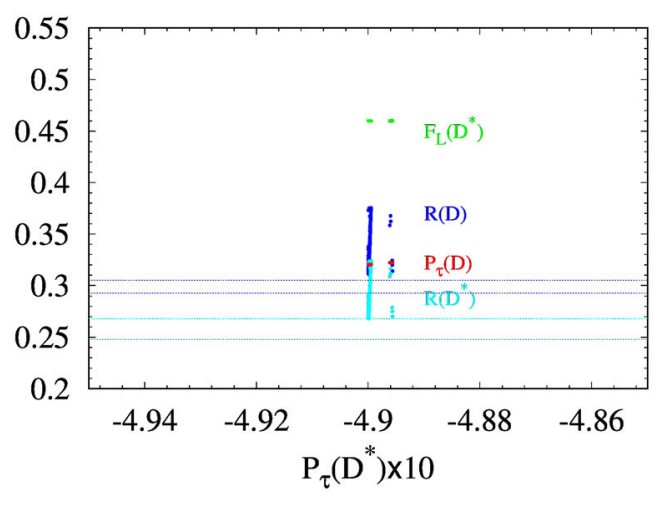

(a)

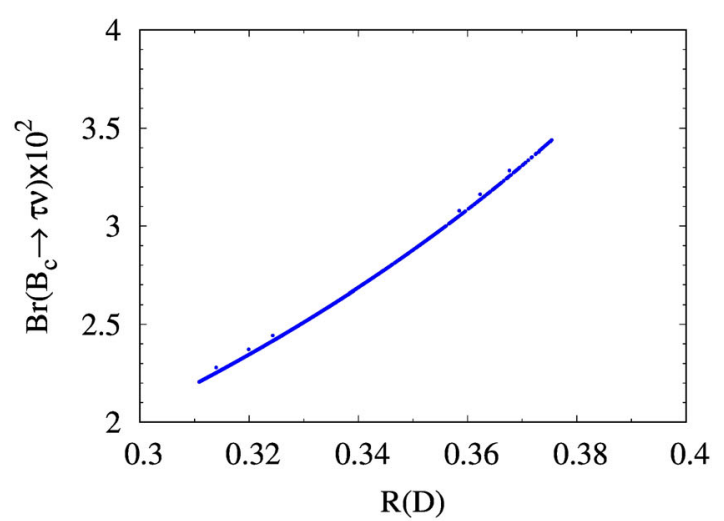

(b)

FIG. 3. Allowed values for various observables at $2 \sigma$. In (a) numerical values of $R\left(D^{(*)}\right), P_{\tau}(D)$, and $F_{L}\left(D^{*}\right)$ are plotted with respect to the values of $P_{\tau}\left(D^{*}\right)$. The horizontal lines are the SM predictions at $2 \sigma$ for $R(D)$ (blue) and $R\left(D^{*}\right)$ (cyan). Other polarization parameters $P_{\tau}\left(D^{(*)}\right)$ and $F_{L}\left(D^{*}\right)$ are consistent with the SM values at $2 \sigma$. In (b) the branching ratio of $\operatorname{Br}\left(B_{c} \rightarrow \tau \nu\right)$ vs $R(D)$ is plotted.

Fig. 3. As can be seen in Fig. 3(a), $R(D)$ is still far away from the SM predictions beyond $2 \sigma$ level, while $R\left(D^{*}\right)$ values have small overlaps at the edge of the SM-allowed range within $2 \sigma$. But the best-fit values of $R\left(D^{(*)}\right)$ in Table I are still beyond the SM by more than $2 \sigma$. Other polarization observables $P_{\tau}\left(D^{(*)}\right)$ and $F_{L}\left(D^{*}\right)$ are consistent with the SM. Figure 3(b) shows that the branching ratio $\operatorname{Br}\left(B_{c} \rightarrow \tau \nu\right)$ lies safely within a few percent.

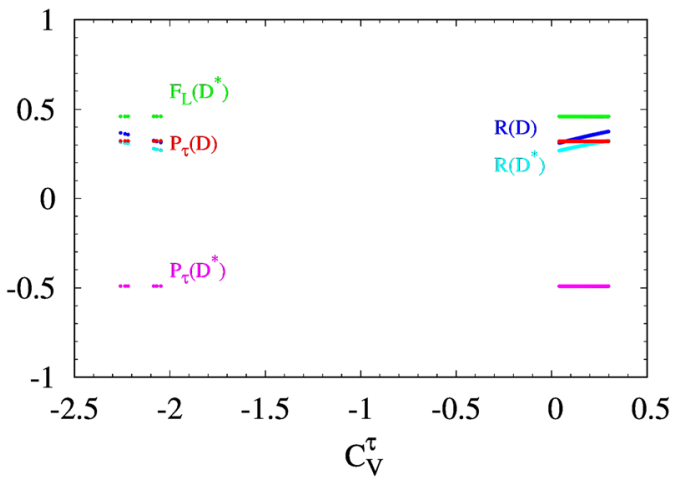

(a)

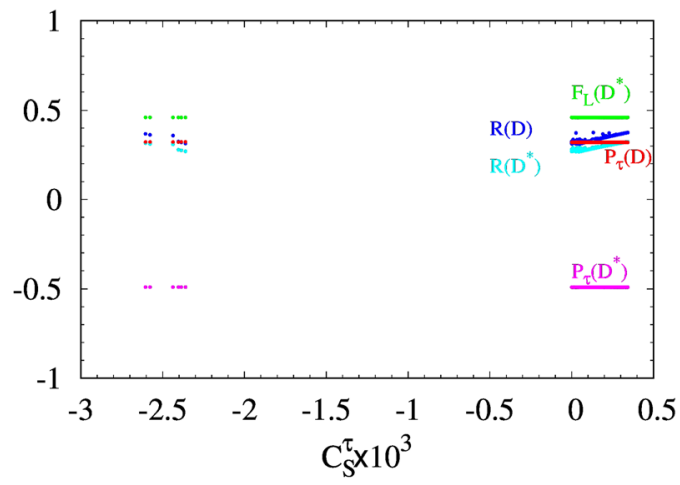

(b)

FIG. 4. Wilson coefficients $C_{V, S}^{\tau}$ and observables. (a) Observables with respect to $C_{V}^{\tau}$; (b) observables with respect to $C_{S}^{\tau} \times 10^{3}$. 


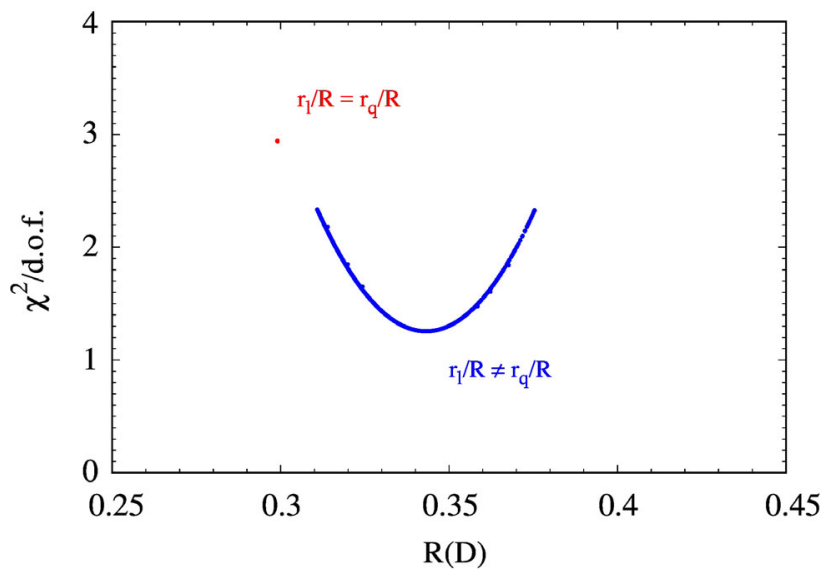

FIG. 5. $R(D)$ vs $\chi^{2} /$ d.o.f. for $r_{\ell} / R=r_{q} / R$ and $r_{\ell} / R \neq r_{q} / R$.

Contributions of the Wilson coefficients to observables at $2 \sigma$ are depicted in Fig. 4. We find that the pattern for $C_{V}^{\mu}$ is very similar to that of $C_{S}^{\mu}$. Note that the Wilson coefficients are

$$
C_{V, S} \sim I_{n}^{q} I_{n}^{\ell},
$$

while the EW precision parameters are

$$
T_{\text {NMUED }}\left(U_{\text {NMUED }}\right) \sim \delta G_{F} \sim\left(I_{n}^{\ell}\right)^{2} .
$$

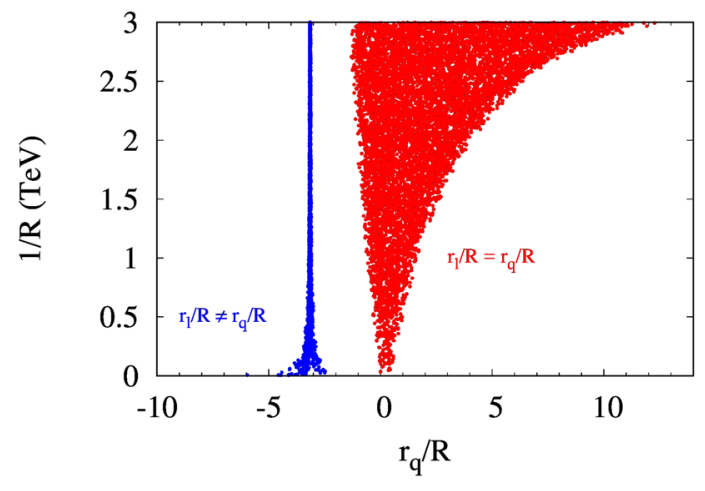

(a)

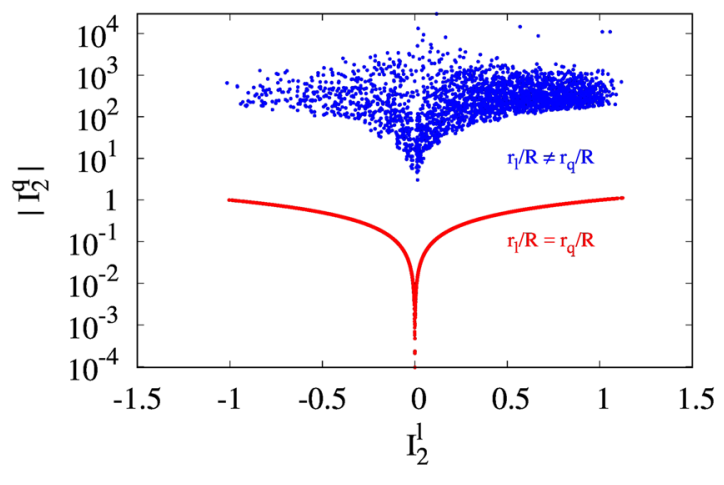

(c)
In the case of $r_{q}=r_{\ell}$ the overlap integrals become $I_{n}^{q}=I_{n}^{\ell}$ and $C_{V, S} \sim\left(I_{n}^{\ell}\right)^{2}$, which are directly affected by the oblique parameters of Eq. (39). According to Eq. (35), EWPT prefers small $\left(I_{n}^{\ell}\right)^{2}$. This means that for $r_{q}=r_{\ell}$ EWPT requires smaller $C_{V, S}$, which results in smaller $R\left(D^{(*)}\right)$ and does not fit the data so well. In other words, we find that $R\left(D^{(*)}\right)$ anomalies require $r_{q} \neq r_{\ell}$ in NMUED. The situation is depicted in Fig. 5, where $R(D)$ vs $\chi^{2} /$ d.o.f. are compared for the $r_{\ell} / R=r_{q} / R$ and $r_{\ell} / R \neq r_{q} / R$ cases.

To see the effects of $r_{\ell} / R \neq r_{q} / R$ more dramatically, we compare the cases of $r_{\ell} / R=r_{q} / R$ and $r_{\ell} / R \neq r_{q} / R$ in Fig. 6. Figure 6(a) shows that the allowed regions of $r_{q} / R$ are quite different from each other. The effect of $r_{\ell} / R \neq r_{q} / R$ appears dramatically on $I_{2}^{q}$, as shown in Figs. 6(b)-6(d). As mentioned above, this is due to the constraints on the oblique parameters. If $r_{q} / R=r_{\ell} / R$, then $I_{2}^{q}=I_{2}^{\ell}$, and it should be kept small to satisfy the EWPT [Fig. 6(b)]. In the case of $r_{q} / R \neq r_{\ell} / R, I_{2}^{q}$ can be very large compared to $I_{2}^{\ell}$ [Fig. 6(c)]. As a result, $R(D)$ is allowed to have large values to fit the data [Fig. 6(d)].

In our analysis $C_{V}^{\tau}=C_{V}^{\mu}$, and we checked the influence of nonzero $C_{V, S}^{\mu}$. Figure 7 shows some of the results. Figure 7(a) depicts $1 / R$ vs $r_{q} / R$, while (b) shows $R(D)$ vs $C_{V}^{\tau}$. We have a figure for $R\left(D^{*}\right)$ similar to Fig. 7(b). Whether or not $C_{V, S}^{\mu}=0$ does not affect the observables

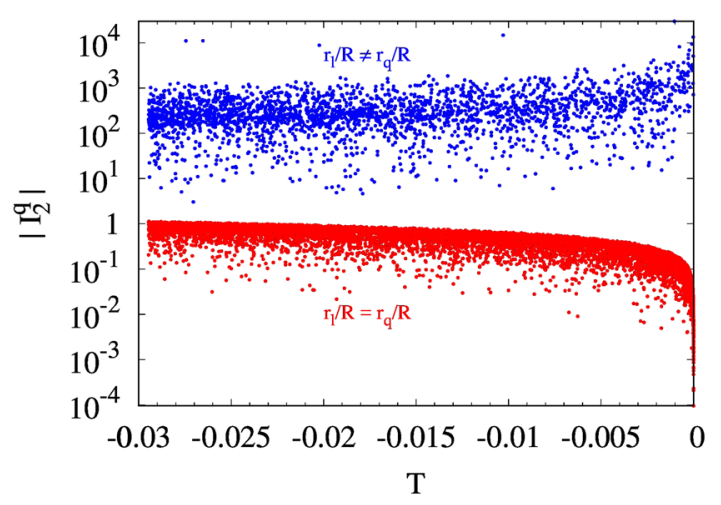

(b)

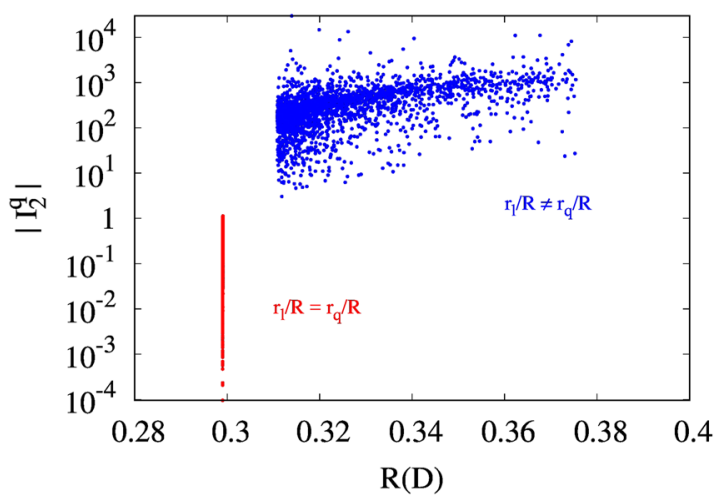

(d)

FIG. 6. Comparisons of various parameter spaces for $r_{q} / R=r_{\ell} / R$ (red) and $r_{q} / R \neq r_{\ell} / R$ (blue) at $2 \sigma$. (a) $1 / R$ (TeV) vs $r_{q} / R$; (b) $\left|I_{2}^{q}\right|$ vs $T ;$ (c) $\left|I_{2}^{q}\right|$ vs $I_{2}^{\ell} ;$ (d) $\left|I_{2}^{q}\right|$ vs $R(D)$. 


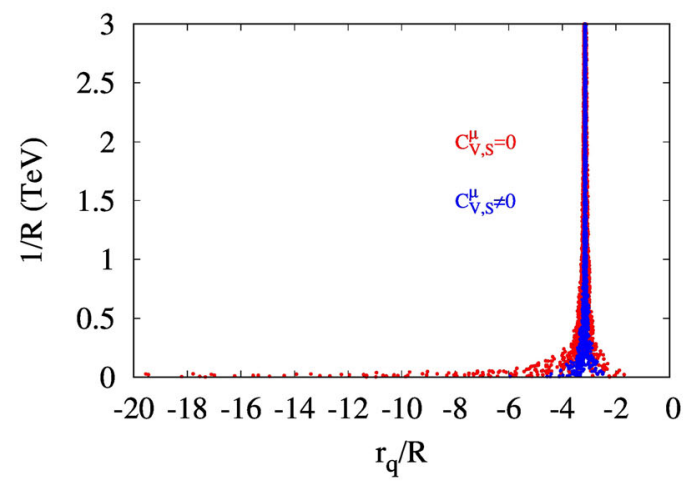

(a)

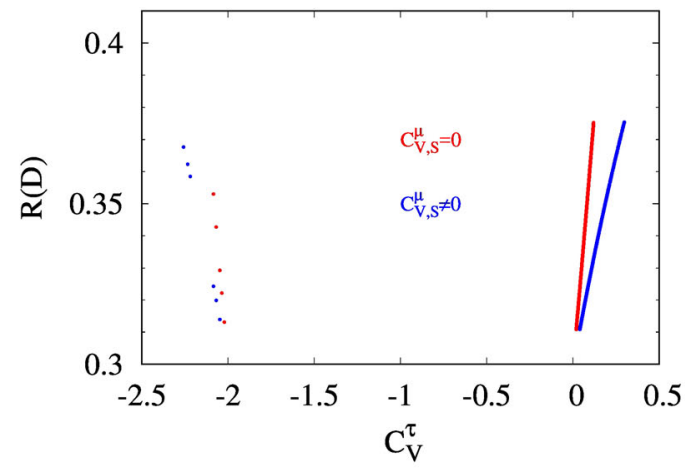

(b)

FIG. 7. Comparisons of parameter spaces for $C_{V, S}^{\mu}=0$ (red) and $C_{V, S}^{\mu} \neq 0$ (blue) at $2 \sigma$. (a) $1 / R$ (TeV) vs $r_{q} / R$; (b) $R(D)$ vs $C_{V}^{\tau}$.

including the polarizations so much, but the allowed range of $r_{q} / R$ or $C_{V}^{\tau}$ could be slightly different. The effect of $C_{S}^{\mu}$ is negligible because its values are very small compared to $C_{S}^{\tau}$. Note that $C_{S}^{\mu}$ is suppressed by $\sim m_{\mu} / m_{\tau}$ with respect to $C_{S}^{\tau}$. And the mixed terms of $\left(1+C_{V}^{\mu, \tau}\right) C_{S}^{\mu, \tau}$ in Eq. (25) are the main source of a difference betweenthe $\tau \nu$ mode and the $\mu \nu$ mode.

In this analysis we do not consider explicitly possible constraints from the flavor changing neutral currents

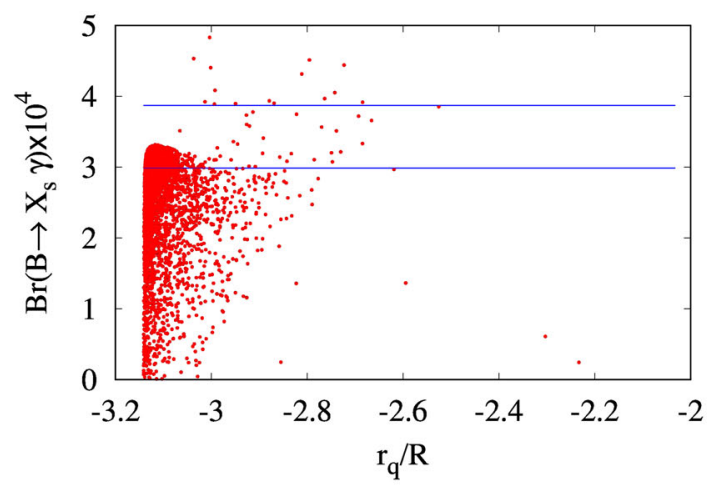

(a)

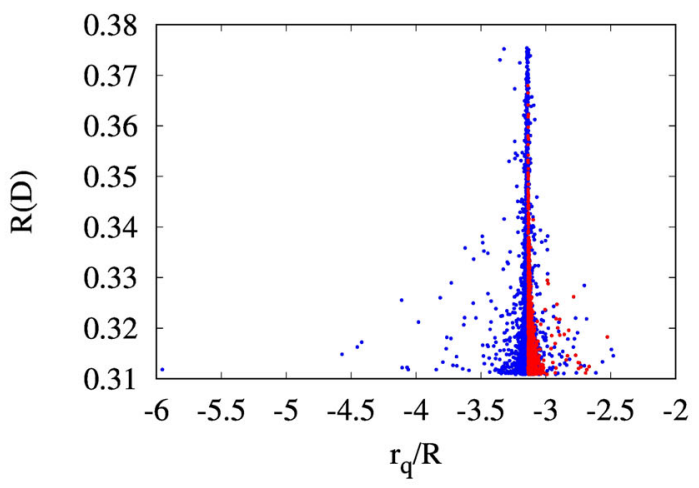

(c)
(FCNC) involving a $b$ quark sector, but it needs some commentary. First, there is no FCNC at tree level because the BLT parameter $r_{q}$ is flavor independent. The effective couplings of the even KK mode of gauge bosons and the SM quarks can be written as the matrix in the flavor space [51]

$$
G^{(n)}=\operatorname{diag}\left(g_{r_{1}}^{X^{(n)}}, g_{r_{2}}^{X^{(n)}}, g_{r_{3}}^{X^{(n)}}\right)
$$

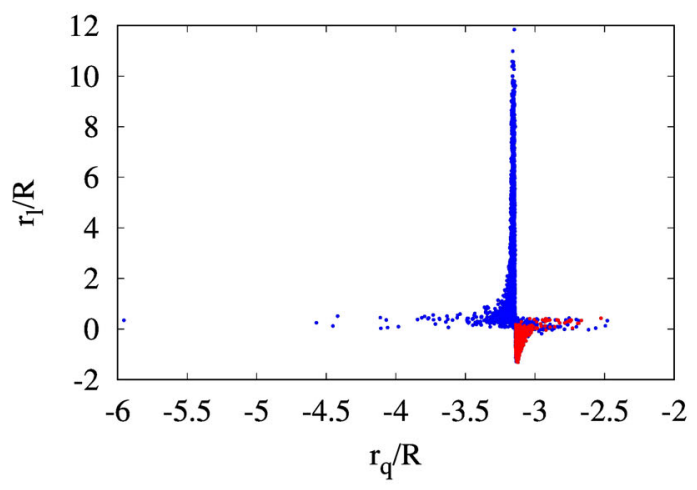

(b)

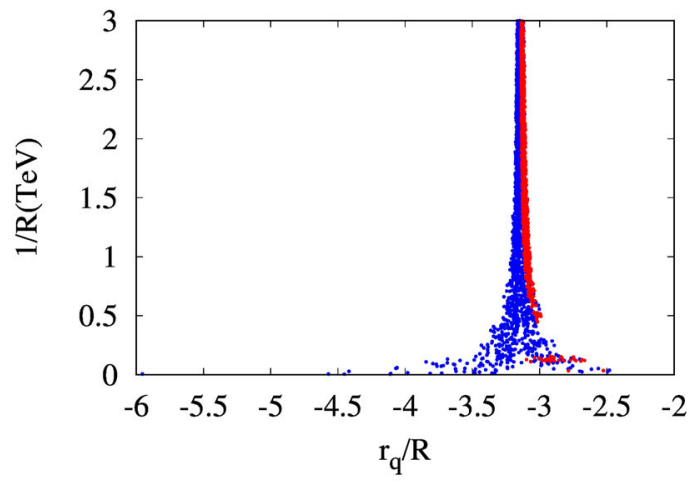

(d)

FIG. 8. Constraints from experimental results of $\operatorname{Br}\left(B \rightarrow X_{s} \gamma\right)=(3.43 \pm 0.21 \pm 0.07) \times 10^{-4}$ [49,66]. (a) Values of $\operatorname{Br}\left(B \rightarrow X_{s} \gamma\right)$ with the points in Fig. 1 but with $-\pi<r_{q} / R$. Blue horizontal lines are $2 \sigma$ allowed bounds. (b)-(d) Regions allowed by $\operatorname{Br}\left(B \rightarrow X_{s} \gamma\right)$ (red) compared with those of Fig. 1 (blue). 
where $X^{(n)}=\gamma^{(n)}, Z^{(n)}$ and $r_{1}=r_{2}=r_{3}=r_{q}$ in our case. The result is that $G^{(n)}$ is proportional to the identity matrix. Second, $B_{s} \rightarrow \mu^{+} \mu^{-}$and $B \rightarrow X_{s} \gamma$ are investigated in Refs. [48,49], respectively. The decay of $B_{s} \rightarrow \mu^{+} \mu^{-}$or $B_{s} \rightarrow X_{s} \ell^{+} \ell^{-}$involves both $I_{n}^{q}$ and $I_{n}^{\ell}$, while $B \rightarrow X_{s} \gamma$ involves $I_{n}^{q}$ only. In the former case since $I_{n}^{q}$ and $I_{n}^{\ell}$ both contribute to the process, one can expect that the constraint on $I_{n}^{\ell}$ would not be as strong as that from the oblique parameters. Actually, in Ref. [48] the analysis was done with $r_{q}=r_{\ell}$. As one can see in Fig. 6 of Ref. [48], the allowed parameter space for small $r_{V}$ is compatible with our results. One point that must be noticed is that the lower limit of $R^{-1}$ is about a few hundred $\mathrm{GeV}$, which varies with $r_{V}$ and $r_{q, \ell}$ (see Table II of Ref. [48]). In the case of $r_{q} \neq r_{\ell}$ the allowed parameter space would be larger. In $B \rightarrow X_{s} \gamma$ only $I_{n}^{q}$ contributes to the process. According to Ref. [49], dominant contribution comes not from $I_{n}^{q}$ but from other overlap integrals, $I_{1,2}^{n}$ [see Eqs. (A10) and (A11) of Ref. [49]]. The integrals contain a factor of $\sqrt{1+r_{q} /(\pi R)}$, and we restrict the range of $r_{q}$ as $-\pi<r_{q} / R$ in considering $\operatorname{Br}\left(B \rightarrow X_{s} \gamma\right)$. In Fig. 8 we show the effects of $\operatorname{Br}\left(B \rightarrow X_{s} \gamma\right)$ on parameter space. We consider only the lowest KK mode contributions for convenience. As shown in Fig. 8(b), positively large values of $r_{\ell} / R$ are not allowed. We find that a considerable amount of parameter space is forbidden, but still the value of $\chi_{\min }^{2}$ is almost the same, and the best-fit values of the observables also remain unchanged. Third, $B_{s}-\bar{B}_{s}$ mixing involves only $I_{n}^{q}$ and could provide a very strong constraint on $r_{q}$. The SM prediction of the mass difference $\Delta M_{s}$ [64],

$$
\Delta M_{s}^{\mathrm{SM}}=(20.01 \pm 1.25) / \mathrm{ps},
$$

is larger by $1.8 \sigma$ than the measured value [1],

$$
\Delta M_{s}^{\exp }=(17.757 \pm 0.021) / \mathrm{ps} .
$$

Usually the NP gives a positive contribution, and $\Delta M_{s}$ puts much stronger bounds on NP than before because the updated SM prediction of $\Delta M_{s}$ gets larger [65]. In our case $\Delta M_{s}$ is roughly $\sim\left(I_{n}^{q}\right)^{4}\left(m_{W} / M_{K K}\right)^{4}$, where $M_{K K}$ is the mass of the mediating KK particle. At $2 \sigma$ level, $\Delta M_{s}^{\exp } /\left(\Delta M_{s}^{\mathrm{SM}}-2 \delta \Delta M_{s}^{\mathrm{SM}}\right)-1 \simeq 0.014$, where $\delta \Delta M_{s}^{\mathrm{SM}}$ is the $1 \sigma$ deviation of $\Delta M_{s}^{\mathrm{SM}}$. One can naively guess that for $M_{K K} \sim 1 \mathrm{TeV}$ only order 1 of $I_{n}^{q}$ is allowed, which could severely constrain $r_{q}$. Further study on this issue is necessary to scrutinize the model.

\section{CONCLUSIONS}

In conclusion, we investigated the $B \rightarrow D^{(*)}$ anomalies in the NMUED model. In the model $n$th KK modes of $W$ boson and scalar couple to a pair of zero-mode fermions to result in nonzero NP Wilson coefficients. We found that the NMUED model successfully fits the current data including $D^{*}$ polarizations, at the sacrifice of $r_{\ell} / R=r_{q} / R$. The EWPT plays a significant role in the model. Our main result is that the enhancement of the overlap integral in the quark sector is very crucial to explaining the $B$ anomalies. If there would be a quite strong constraint on the quark sector (e.g., from the neutral meson mixing or whatever), then it could restrict the validity of the NMUED model seriously. We also found that the branching ratio $\operatorname{Br}\left(B_{c} \rightarrow \tau \nu\right)$ stays at a few percent, well below $10 \%$. In our analysis $R(D)$ values have no overlap with the SM predictions at $2 \sigma$ level, while $R\left(D^{*}\right)$ touches the SM-allowed region. Future measurements of more observables would check further the validity of the NMUED model.
[1] Y. Amhis et al. (HFLAV Collaboration), Eur. Phys. J. C 77, 895 (2017).

[2] J. P. Lees et al. (BABAR Collaboration), Phys. Rev. Lett. 109, 101802 (2012).

[3] J. P. Lees et al. (BABAR Collaboration), Phys. Rev. D 88, 072012 (2013).

[4] M. Huschle et al. (Belle Collaboration), Phys. Rev. D 92, 072014 (2015).

[5] Y. Sato et al. (Belle Collaboration), Phys. Rev. D 94, 072007 (2016).

[6] S. Hirose (Belle Collaboration), Nucl. Part. Phys. Proc. 287-288, 185 (2017).

[7] S. Hirose et al. (Belle Collaboration), Phys. Rev. Lett. 118, 211801 (2017).

[8] S. Hirose et al. (Belle Collaboration), Phys. Rev. D 97, 012004 (2018).
[9] A. Abdesselam et al. (Belle Collaboration), arXiv:1904 .08794 .

[10] R. Aaij et al. (LHCb Collaboration), Phys. Rev. Lett. 115, 111803 (2015); 115, 159901(E) (2015).

[11] R. Aaij et al. (LHCb Collaboration), Phys. Rev. D 97, 072013 (2018).

[12] Average of $R(D)$ and $R\left(D^{*}\right)$ for Spring 2019, Heavy Flavor Averaging Group, https://hflav-eos.web.cern.ch/hflav-eos/ semi/spring19/html/RDsDsstar/RDRDs.html.

[13] J. P. Lee, Phys. Rev. D 96, 055005 (2017).

[14] A. Crivellin, C. Greub, and A. Kokulu, Phys. Rev. D 86, 054014 (2012); 87, 094031 (2013); A. Crivellin, J. Heeck, and P. Stoffer, Phys. Rev. Lett. 116, 081801 (2016).

[15] P. Biancofiore, P. Colangelo, and F. De Fazio, Phys. Rev. D 87, 074010 (2013).

[16] J. M. Cline, Phys. Rev. D 93, 075017 (2016). 
[17] M. A. Ivanov, J. G. Körner, and C. T. Tran, Phys. Rev. D 94, 094028 (2016); 95, 036021 (2017).

[18] C. H. Chen and T. Nomura, Eur. Phys. J. C 77, 631 (2017).

[19] S. Iguro and K. Tobe, Nucl. Phys. B925, 560 (2017).

[20] I. Doršner, S. Fajfer, N. Košnik, and I. Nišandžić, J. High Energy Phys. 11 (2013) 084.

[21] R. Alonso, B. Grinstein, and J. Martin Camalich, J. High Energy Phys. 10 (2015) 184.

[22] M. Bauer and M. Neubert, Phys. Rev. Lett. 116, 141802 (2016).

[23] R. Barbieri, G. Isidori, A. Pattori, and F. Senia, Eur. Phys. J. C 76, 67 (2016).

[24] L. Di Luzio, A. Greljo, and M. Nardecchia, Phys. Rev. D 96, 115011 (2017).

[25] L. Calibbi, A. Crivellin, and T. Li, Phys. Rev. D 98, 115002 (2018).

[26] D. Bečirević, I. Doršner, S. Fajfer, N. Košnik, D. A. Faroughy, and O. Sumensari, Phys. Rev. D 98, 055003 (2018).

[27] R. Barbieri, C. W. Murphy, and F. Senia, Eur. Phys. J. C 77, 8 (2017).

[28] D. Buttazzo, A. Greljo, G. Isidori, and D. Marzocca, J. High Energy Phys. 11 (2017) 044.

[29] M. Bordone, C. Cornella, J. Fuentes-Martin, and G. Isidori, Phys. Lett. B 779, 317 (2018).

[30] S. Matsuzaki, K. Nishiwaki, and K. Yamamoto, arXiv: 1903.10823.

[31] E. Megias, M. Quiros, and L. Salas, J. High Energy Phys. 07 (2017) 102.

[32] E. Megias, M. Quiros, and L. Salas, Phys. Rev. D 96, 075030 (2017).

[33] G. D'Ambrosio and A. M. Iyer, Eur. Phys. J. C 78, 448 (2018).

[34] M. Blanke and A. Crivellin, Phys. Rev. Lett. 121, 011801 (2018).

[35] X. W. Kang, T. Luo, Y. Zhang, L. Y. Dai, and C. Wang, Eur. Phys. J. C 78, 909 (2018).

[36] Z. R. Huang, Y. Li, C. D. Lu, M. A. Paracha, and C. Wang, Phys. Rev. D 98, 095018 (2018).

[37] D. Bardhan and D. Ghosh, Phys. Rev. D 100, 011701 (2019).

[38] M. Tanaka and R. Watanabe, Phys. Rev. D 82, 034027 (2010).

[39] M. Tanaka and R. Watanabe, Phys. Rev. D 87, 034028 (2013).

[40] A. Abdesselam et al. (Belle Collaboration), arXiv:1903 .03102 .

[41] A. K. Alok, D. Kumar, S. Kumbhakar, and S. U. Sankar, Phys. Rev. D 95, 115038 (2017).
[42] H. C. Cheng, K. T. Matchev, and M. Schmaltz, Phys. Rev. D 66, 036005 (2002).

[43] F. del Aguila, M. Perez-Victoria, and J. Santiago, Acta Phys. Pol. B 34, 5511 (2003).

[44] F. del Aguila, M. Perez-Victoria, and J. Santiago, J. High Energy Phys. 02 (2003) 051.

[45] T. Flacke, A. Menon, and D. J. Phalen, Phys. Rev. D 79, 056009 (2009).

[46] A. Datta, U. K. Dey, A. Shaw, and A. Raychaudhuri, Phys. Rev. D 87, 076002 (2013).

[47] A. Datta and A. Shaw, Mod. Phys. Lett. A 31, 1650181 (2016).

[48] A. Datta and A. Shaw, Phys. Rev. D 93, 055048 (2016).

[49] A. Datta, and A. Shaw (Indian Association for the Cultivation of Science Collaboration), Phys. Rev. D 95, 015033 (2017).

[50] A. Biswas, A. Shaw, and S. K. Patra, Phys. Rev. D 97, 035019 (2018).

[51] S. Dasgupta, U. K. Dey, T. Jha, and T. S. Ray, Phys. Rev. D 98, 055006 (2018).

[52] T. Jha and A. Datta, J. High Energy Phys. 03 (2015) 012.

[53] M. Blanke, A. Crivellin, S. de Boer, T. Kitahara, M. Moscati, U. Nierste, and I. Nišandžić, Phys. Rev. D 99, 075006 (2019).

[54] S. Aoki et al., Eur. Phys. J. C 77, 112 (2017).

[55] F. U. Bernlochner, Z. Ligeti, M. Papucci, and D. J. Robinson, Phys. Rev. D 95, 115008 (2017); 97, 059902(E) (2018).

[56] R. Alonso, B. Grinstein, and J. Martin Camalich, Phys. Rev. Lett. 118, 081802 (2017).

[57] A. G. Akeroyd and C. H. Chen, Phys. Rev. D 96, 075011 (2017).

[58] T. Flacke and C. Pasold, Phys. Rev. D 85, 126007 (2012).

[59] T. Flacke, K. Kong, and S. C. Park, J. High Energy Phys. 05 (2013) 111.

[60] A. Datta, K. Nishiwaki, and S. Niyogi, J. High Energy Phys. 01 (2014) 104.

[61] U. K. Dey and T. Jha, Phys. Rev. D 94, 056011 (2016).

[62] M. Baak, J. Cúth, J. Haller, A. Hoecker, R. Kogler, K. Mönig, M. Schott, J. Stelzer (Gfitter Group), Eur. Phys. J. C 74, 3046 (2014).

[63] T. Flacke, D. W. Kang, K. Kong, G. Mohlabeng, and S. C. Park, J. High Energy Phys. 04 (2017) 041.

[64] L. Di Luzio, M. Kirk, and A. Lenz, Phys. Rev. D 97, 095035 (2018).

[65] L. Di Luzio, M. Kirk, and A. Lenz, arXiv:1811.12884.

[66] Y. Amhis et al. (Heavy Flavor Averaging Group), arXiv: 1412.7515. 